\title{
Lagrangian intersections, critical points and Qcategory
}

\author{
P.-M. MOYAUX L. VANDEMBROUCQ
}

U.F.R. de Mathématiques, Université de Lille 1, 59655 Villeneuve d'Ascq, FRANCE, moyaux@agat.univ-lille1.fr

CMAT, Departamento de Matemática, Universidade do Minho, Campus de Gualtar, 4710 Braga,PORTUGAL, lucile@math.uminho.pt

\begin{abstract}
For a manifold $M$, we prove that any function defined on a vector bundle of basis $M$ and quadratic at infinity has at least $Q \operatorname{cat}(M)+1$ critical points. Here $Q \operatorname{cat}(M)$ is a homotopically stable version of the LS-category defined by Scheerer, Stanley and Tanré [27]. The key homotopical result is that $Q \operatorname{cat}(M)$ can be identified with the relative LS-category of Fadell and Husseini [9] of the pair $\left(M \times D^{n+1}, M \times S^{n}\right)$ for $n$ big enough.

Combining this result with the work of Laudenbach and Sikorav [19], we obtain that if $M$ is closed, for any hamiltonian diffeomorphism with compact support $\psi$ of $T^{*} M, \#(\psi(M) \cap M) \geq Q \operatorname{cat}(M)+1$, which improves all previously known homotopical estimates of this intersection number.
\end{abstract}

Mathematics Subject Classification (2000) : 53D12, 55M30, 57R70

\section{Introduction}

The homotopy type of a manifold and the dynamics of the maps it can support are closely related. For example, let $M$ be a manifold and consider $\operatorname{crit}(M)$, the least number of critical points of a function $f: M \rightarrow \mathbf{R}$ (constant maximal and regular on $\partial M$ if $\partial M \neq \emptyset)$. The computation of $\operatorname{crit}(M)$ is usually very difficult. For example, it is still unknown whether crit(-) is a homotopy invariant. Indeed, a positive answer to this problem would solve the Poincaré conjecture [31], since it would tell us that a manifold with the homotopy type of a sphere supports a map with exactly two critical points and thus is homeomorphic to the sphere. However, it is possible to study $\operatorname{crit}(M)$ through its approximations by homotopy invariants. The first of these approximations is the Lusternik-Schnirelmann category. For a space $X$, recall that $\operatorname{cat}(X)$ is the smallest $n$ such that $X$ may be covered by $n+1$ open sets contractible in $X$. Lusternik and Schnirelmann proved that if $M$ is a closed manifold,

$$
\operatorname{crit}(M) \geq \operatorname{cat}(M)+1
$$

This inequality is also valid for manifold with boundaries [31]. Remark that in both cases, $1+\operatorname{cat}(M)$ is the best homotopical lower bound known for $\operatorname{crit}(M)$. 
Despite the apparent simplicity of its definition, the category is usually very hard to compute. Hence, equivalent definitions of the category have been sought. In particular, $\operatorname{cat}(X)$ may also be defined as the smallest $n$ such that the inclusion of the $n^{\text {th }}$ stage of Milnor classifying construction of $B \Omega X$, $j_{n}: B_{n}(\Omega X) \hookrightarrow B \Omega X$ has a section. Notice also that many approximations of the category have been defined by applying functors to the maps $j_{n}$ and looking for sections to the maps thus obtained. Central to our purpose are the $Q^{p}$ - and the $Q$ category of Scheerer, Stanley, Tanré [27]. These are invariants associated by the above process to the fiberwise extension of the functors $\Omega^{p} \Sigma^{p}$ and $\Omega^{\infty} \Sigma^{\infty}$. We will also use the invariant associated to the functor $\Sigma^{\infty}$ called $\sigma$ category as in [32], which also coincides with the invariant $r(M)$ of [24].

It is also possible to obtain critical points estimates for functions on $\partial$-manifolds having on the boundary a more complicated behavior that the one described in [31]. For example, if $M$ is a closed manifold, consider the functions defined on $M \times D^{n}$ and with exit set for the associated flow $M \times D^{n-t-1} \times S^{t} \subset \partial\left(M \times D^{n}\right)$ (see definition 5.2 in [25]). These functions are important in symplectic geometry [2] and it is interesting to estimate their number of critical points. In particular, using a relative version of the LS-category due to Fadell and Husseini [9], Cornea [3] proves that such functions have at least $\operatorname{cat}\left(M \times D^{t+1}, M \times S^{t}\right)$ critical points. Another estimate is obtained by Rudyak [25], who proves that these functions have at least $\sigma c a t(M)+1$ critical points.

Our work is close in spirit to the works of Cornea and Rudyak mentionned above. Indeed, we prove

Theorem A. Let $M$ be a manifold. Then,

$$
\widetilde{\operatorname{crit}}(M) \geq \inf _{n \in \mathbf{N}} \operatorname{cat}\left(M \times D^{n+1}, M \times S^{n}\right)=\operatorname{Qcat}(M)+1
$$

where $\widetilde{\operatorname{crit}}(M)$ is the least number of critical points of a function quadratic at infinity over $M$ ( satisfying a boundary condition if $\partial M \neq \emptyset$ ). This inequality may be seen as a "stable" version of the Lusternik-Schnirelmann Theorem which provides a link between the notions of homotopical and dynamical stabilizations. Indeed, the class of functions quadratic at infinity over $M$ contains the dynamical suspensions of functions on $M$. Theorem A gives an estimate of the number of critical points of these functions in terms of a stable version (in the homotopical sense) of the category of $M$.

Remember also that the functions quadratic at infinity are central in the problem of lagrangian intersections in cotangent bundles. Indeed, one of the forms of the Arnold conjecture may be stated as

Arnold conjecture : Let $M$ be a closed manifold. For any hamiltonian diffeomorphism with compact support $\psi$ of $T^{*} M$,

$$
\#(\psi(M) \cap M) \geq \operatorname{crit}(M)
$$

This conjecture has motivated a lot of work. In particular, Hofer proved in 1985 that $\#(\psi(M) \cap M) \geq \cup$-length $(M)+1$. The same year, Laudenbach and 
Sikorav [19] proved the inequality $\#(\psi(M) \cap M) \geq \widetilde{\operatorname{crit}}(M)$. Combining this last result with Theorem $A$, we obtain :

Corollary B. Let $M$ be a closed manifold. For any hamiltonian diffeomorphism with compact support $\psi$ of $T^{*} M$,

$$
\#(\psi(M) \cap M) \geq \operatorname{Qcat}(M)+1
$$

This is the best homotopical lower bound known for this intersection number. For example, since $\operatorname{Qcat}(M) \geq \cup$-length $(M)$, we obtain the inequality of [16] as corollary. Notice also that the strict inequality $\operatorname{Qcat}(M)>\cup$-length $(M)$ may occur. For example, we will see in section 3.1 that this is the case for $S p(2)$ $(3>2)$ and $S p(3)(5>3)$. Moreover, there are very few examples of closed manifolds with Qcat $\neq$ cat. Indeed (see section 3), it is conjectured in [27] that such manifolds are exactly the counter-examples to the Ganea conjecture and very few of them are known. Remark for example that for all manifolds $M=N \times T^{n}$ with $N$ a closed manifold, $n \geq \operatorname{dim}(N)+3$ and $T^{n}$ the $n$-Torus, we have $Q \operatorname{cat}(M)=\operatorname{cat}(M)$.

Remark at last that combining the equality in Theorem $A$ and Cornea estimate, we obtain that the functions on $M \times D^{n}$ with the boundary behavior described above have at least $Q \operatorname{cat}(M)+1$ critical points. Since $Q \operatorname{cat}(M) \geq \sigma \operatorname{cat}(M)$ (see section 3.1), we obtain the estimate in [25] as corollary.

The proof of Theorem $A$ splits in two parts. At first, using critical points estimates, we give a link between $\widetilde{\operatorname{crit}}(M)$ and the category of the pairs $(M \times$ $\left.D^{n+1}, M \times S^{n}\right)$. Afterwards, we link together the invariants $\operatorname{cat}\left(M \times D^{n+1}, M \times\right.$ $\left.S^{n}\right)$ and $Q \operatorname{cat}(M)$. In the last section of this paper, we discuss some consequences of Theorem A for the Arnold Conjecture, for the computation of $\operatorname{crit}(M)$ and for the study of others conjectures on numerical invariants. In particular, we notice that, as a consequence of results in [4], for simply-connected manifolds we also have the inequality $\operatorname{cat}(M)+2 \geq \widetilde{\operatorname{crit}}(M)$.

Throughout this paper, we work in the category of compactly generated spaces having the homotopy type of $C W$-complexes (this category will be denoted by $\mathcal{T}$ op). All manifolds are assumed to be smooth, riemannian manifolds.

\section{Proof of Theorem A}

The Theorem A is an immediate consequence of Corollaries 1 and 2 below.

\subsection{Relative LS category and critical points}

Definition 1 Let $(X, A)$ be a pair of spaces, with $A \hookrightarrow X$ a cofibration. Then, $\operatorname{cat}(X, A)$ is the smallest $k$ such that $X$ may be covered by $k+1$ open sets $\left(U_{i}\right)_{0 \leq i \leq k}$ such that $U_{0}$ can be deformed into $A$ (rel. A) and the other open sets are contractible in $X$.

As its absolute counterpart, the relative category may be used as lower bound for critical points estimates.

In particular, we recall the following result, due to Cornea [3]. 
Let $M$ be a compact, riemannian manifold and $f: M \rightarrow \mathbf{R}$ a smooth function. Let $\left(N_{1}, N_{0}\right)$ be a pair of subspaces of $M$ satisfying :

- $N_{1}$ is a manifold with boundary, of same dimension as $M$, and $N_{0}$ is the part of $\partial N_{1}$ where $-\nabla f$ points outward;

- $f$ has no critical point on $\partial N_{1}$.

Then, $f$ has at least $\operatorname{cat}\left(N_{1}, N_{0}\right)$ critical points strictly inside $N_{1}$.

Definition 2 Let $M$ be a riemannian manifold and $\nu=(\pi ; E ; M)$ a vector bundle over $M$. Endow $E$ with a metric which is locally a product metric of the metric on $M$ with the canonical metric on $\mathbf{R}^{n}$.

- A smooth function $q: E \rightarrow \mathbf{R}$ is a non degenerate quadratic form of index $i$ over $M$ if the restriction of $q$ to each fiber is (modulo a constant depending on the point of the base) a non degenerate quadratic form of index $i$.

If moreover $q_{\mid M}=0$, then $q$ is said to be strict.

- A smooth function $f: E \rightarrow \mathbf{R}$ is quadratic at infinity of index $i$ over $M$ if $f$ coincides, outside of a compact neighborhood of the zero section, with a non degenerate quadratic form of index $i$.

Remark 1 i) A quadratic form over $M$ can be seen as the sum of a function defined on $M$ with a strict quadratic form over $M$.

ii) If $M$ is a closed manifold, then any function quadratic at infinity over $M$ and defined on a trivial bundle is a function as in [3], [25]. Moreover, in the following proof, if $f$ is quadratic at infinity over $M$, we construct another function quadratic at infinity over $M$, defined on a trivial bundle and with the same number of critical points as the initial function.

In the following, we restrict ourselves to functions quadratic at infinity over $M$ satisfying (with the above notations) the following boundary condition.

$\mathcal{C}: \nabla f$ points outward on $\partial E$. In particular, $f$ has no critical points on $\partial E$. Of course, if $M$ is closed then condition $\mathcal{C}$ is empty. We define now :

Definition $3 \widetilde{\operatorname{crit}}(M)$ is the least number of critical points of a function quadratic at infinity over $M$ satisfying $\mathcal{C}$.

Proposition 1 Let $M$ be a connected manifold of dimension $n, \nu=(\pi ; E$;

$M)$ be a vector bundle and $f: E \rightarrow \mathbf{R}$ a function quadratic at infinity over $M$ of index $i$ and satisfying condition $\mathcal{C}$. We have

$$
\operatorname{crit}(f) \geq \operatorname{cat}\left(M \times D^{n+i}, M \times S^{n+i-1}\right)
$$

Proof. In this proof, 'form' or 'quadratic form' stands for 'non degenerate quadratic form'. With the above notations, set $r=\operatorname{rank}(\nu)$. Fix an orthogonal structure on $\nu$.

Let $K$ be a compact neighborhood of the zero section in $E$ such that $f$ equals a quadratic form $q$ outside of $K$. We may assume without loss of generality that $q$ also satisfies $\mathcal{C}$. Indeed, $q$ satisfies $\mathcal{C}$ except maybe inside of $K$. Now, consider a function $g: M \rightarrow \mathbf{R}$ which is zero except on a collar of $\partial M$ where its gradient points outward. It is possible to choose $g$ (the choice of the collar depending on $f$, the choice of $\|\nabla g\|$ depending on $q$ ) such that the quadratic form $q+g \circ \pi$ satisfies $\mathcal{C}$ and $\operatorname{crit}(f+g \circ \pi)=\operatorname{crit}(f)$. 
Il follows from [20], lemma 3 , that we may restrict ourselves to the case where there is a splitting $E_{u} \bigoplus E_{s} \cong E$ such that $q(u \oplus v)=q_{u}(u)+q_{s}(v)$ with $q_{u}: E_{u} \rightarrow \mathbf{R}$ a positive form and $q_{s}: E_{s} \rightarrow \mathbf{R}$ a negative form. Notice that $\operatorname{rank}\left(\nu_{u}\right)=r-i$ and $\operatorname{rank}\left(\nu_{s}\right)=i$.

Take $\nu_{s}^{\prime}$, the inverse bundle of $\nu_{s}$ such that $E_{s} \bigoplus E_{s}^{\prime} \cong M \times \mathbf{R}^{n+i}$ and let $q_{s}^{\prime}$ be a strict negative quadratic form on $E_{s}^{\prime}$ with gradient on $\partial E_{s}^{\prime}$ tangent to $\partial E_{s}^{\prime}$. (Such a function is easy to construct. Consider a negative strict quadratic form on $\partial E_{s}^{\prime}$ over $\partial M$ and extend it on a collar of $\partial E_{s}^{\prime}$ in $E_{s}^{\prime}$. By means of a partition of unity, it is easy to glue this function with a negative strict quadratic form on $E_{s}^{\prime}$ in such a way to get a negative strict quadratic form over $M$ with the required property.) The form $Q_{s}=q_{s} \oplus q_{s}^{\prime}$ may be seen as a negative quadratic form on $M \times \mathbf{R}^{n+i}$.

Similarly, take $\nu_{u}^{\prime}$ such that $E_{u}^{\prime} \bigoplus E_{u} \cong M \times \mathbf{R}^{n+r-i}$ and let $q_{u}^{\prime}$ be a positive strict quadratic form on $E_{u}^{\prime}$ with gradient on $\partial E_{u}^{\prime}$ tangent to $\partial E_{u}^{\prime}$. The form $Q_{u}=q_{u}^{\prime} \oplus q_{u}$ may be seen as a positive quadratic form on $M \times \mathbf{R}^{n+r-i}$.

Thus, $Q=q_{u}^{\prime} \oplus q \oplus q_{s}^{\prime}=q_{u}^{\prime} \oplus q_{u} \oplus q_{s} \oplus q_{s}^{\prime}=Q_{u} \oplus Q_{s}$ is a quadratic form of index $n+i$ on $E_{u}^{\prime} \bigoplus E_{u} \bigoplus E_{s} \bigoplus E_{s}^{\prime} \cong M \times \mathbf{R}^{n+r-i} \times \mathbf{R}^{n+i} \cong M \times \mathbf{R}^{2 n+r}$. Moreover, $\nabla Q$ points outward on $\partial M \times \mathbf{R}^{2 n+r}$.

Now, we construct a function $F$ on $E_{u}^{\prime} \bigoplus E_{u} \bigoplus E_{s} \bigoplus E_{s}^{\prime}$ such that $\operatorname{crit}(F)=$ $\operatorname{crit}(f), F$ is equal to $Q$ outside of a compact neighborhood of the zero section in $M \times \mathbf{R}^{2 n+r}$ and $F$ satisfies condition $\mathcal{C}$.

The strict quadratic forms $q_{u}^{\prime}$ and $q_{s}^{\prime}$ are non degenerate and thus have 0 as unique critical point in each fiber. Thus, clearly, $\operatorname{crit}(f)=\operatorname{crit}\left(q_{u}^{\prime} \oplus f \oplus q_{s}^{\prime}\right)$.

Now, let $\lambda: E_{u}^{\prime} \bigoplus E_{s}^{\prime} \rightarrow[0 ; 1]$ be a smooth function such that $\lambda\left(u^{\prime}, v^{\prime}\right)=0$ if $\left\|u^{\prime}\right\|^{2}+\left\|v^{\prime}\right\|^{2} \leq 1$ and $\lambda\left(u^{\prime}, v^{\prime}\right)=1$ if $\left\|u^{\prime}\right\|^{2}+\left\|v^{\prime}\right\|^{2} \geq 2$ and with gradient tangent to $\partial\left(E_{u}^{\prime} \bigoplus E_{s}^{\prime}\right)$.

Define $F\left(u^{\prime}, u, v, v^{\prime}\right)=q_{u}^{\prime}\left(u^{\prime}\right)+\left(f(u, v)+\lambda\left(u^{\prime}, v^{\prime}\right)(q(u, v)-f(u, v))\right)+q_{s}^{\prime}\left(v^{\prime}\right)$.

Thus, $F=q_{u}^{\prime} \oplus f \oplus q_{s}^{\prime}$ near the zero section and $F=Q$ outside of a compact neighborhood $K^{\prime}$ of the zero section. Possibly changing $q_{u}^{\prime}$ and $q_{s}^{\prime}$ (multiplication by positive constants), we may ensure that $F$ has no critical points if $1 \leq\left\|u^{\prime}\right\|^{2}+\left\|v^{\prime}\right\|^{2} \leq 2$, and thus no critical points if $\left\|u^{\prime}\right\|^{2}+\left\|v^{\prime}\right\|^{2} \geq 1$.

This quickly leads to $\operatorname{crit}(F)=\operatorname{crit}\left(q_{u}^{\prime} \oplus f \oplus q_{s}^{\prime}\right)=\operatorname{crit}(f)$ and all the critical points of $F$ are enclosed in $K^{\prime}$. Remark also that $F$ clearly satisfies condition $\mathcal{C}$.

Now, choose the disks $D^{n+r-i}$ and $D^{n+i}$ such that $K^{\prime} \subset M \times D^{n+r-i} \times D^{n+i}$. Clearly, the pair $\left(M \times D^{n+r-i} \times D^{n+i}, M \times D^{n+r-i} \times S^{n+i-1}\right)$ satisfies the hypothesis of Cornea result for the flow induced by $-\nabla Q$ and thus, for the flow induced by $-\nabla F$.

It follows that $F$ has at least $\operatorname{cat}\left(M \times D^{n+r-i} \times D^{n+i}, M \times D^{n+r-i} \times S^{n+i-1}\right)$ critical points.

Thus, $\operatorname{crit}(f)=\operatorname{crit}(F) \geq \operatorname{cat}\left(M \times D^{n+r-i} \times D^{n+i}, M \times D^{n+r-i} \times S^{n+i-1}\right)=$ $\operatorname{cat}\left(M \times D^{n+i}, M \times S^{n+i-1}\right)$.

From this result, we deduce easily : 
Corollary 1 For any manifold $M$,

$$
\widetilde{\operatorname{crit}}(M) \geq \inf _{n \in \mathbf{N}} \operatorname{cat}\left(M \times D^{n+1}, M \times S^{n}\right)
$$

\subsection{Relative category and fiberwise suspension construc- tion}

\subsubsection{The Ganea Fibrations}

In the following, we assume all the spaces to be well-pointed.

We give now another definition of the relative and absolute categories.

First, recall that any map $f: A \rightarrow X$ may be decomposed as a homotopy equivalence followed by a fibration :

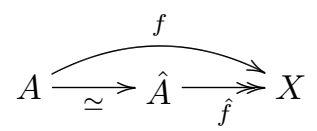

where $\hat{A}=\left\{(a, \omega) \in A \times X^{I} / f(a)=\omega(0)\right\}, \hat{f}(a, \omega)=\omega(1)$ and the map $A \rightarrow \hat{A}$ is given by $a \mapsto(a$, constant path on $f(a))$. This map is clearly a homotopy equivalence with homotopy inverse $\hat{A} \rightarrow A$ given by $(a, \omega) \mapsto a$.

We say that $\hat{f}$ is obtained by turning $f$ into a fibration and the fiber $F$ of $\hat{f}$ is the homotopy fiber of $f$.

Now, using the Fiber-Cofiber construction (see [13], [3]), we define inductively the Ganea Fibrations associated to $f$

$$
F_{n}(X, A) \stackrel{i_{n}(X, A)}{\longrightarrow} G_{n}(X, A) \stackrel{g_{n}(X, A)}{\longrightarrow} X
$$

by :

- $g_{0}(X, A)$ is obtained by turning $f$ into a fibration;

- $g_{n+1}(X, A)$ is obtained by turning the map $g_{n}(X, A) \cup *: G_{n}(X, A) \cup$ $C F_{n}(X, A) \rightarrow X$ into a fibration.

Notice also that, by [14] Thm 1.1, $F_{n+1}(X, A) \simeq F_{n}(X, A) * \Omega X$.

We obtain the diagram

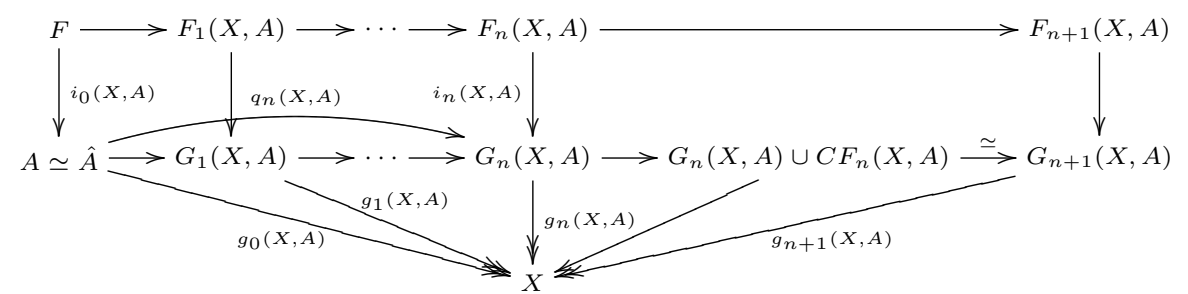

Now, define $q_{n}(X, A)$ as the canonical map $A \rightarrow G_{n}(X, A)$. We have $([3]):$

$$
\begin{aligned}
\operatorname{cat}(X, A) \leq n & \exists s: X \rightarrow G_{n}(X, A) \text { s.t. }\left\{\begin{array}{l}
g_{n}(X, A) \circ s \simeq 1_{X} \\
s \circ f \simeq q_{n}(X, A)
\end{array}\right. \\
& \begin{array}{l}
g_{n}(X ; A):\left(G_{n}(X, A), A\right) \rightarrow(X, A), \text { as a map of pairs, } \\
\text { has a homotopy section }
\end{array}
\end{aligned}
$$


Let $X$ be a space and take $f: P X \rightarrow X$, the path-loop fibration over $X$. In this case, the fibrations obtained by applying the Fiber-Cofiber construction to $f$ are called the Ganea fibrations associated to $X$ and are denoted by

$$
F_{n}(X) \stackrel{i_{n}(X)}{\longrightarrow} G_{n}(X) \stackrel{g_{n}(X)}{\longrightarrow} X
$$

Notice that $g_{n}(X)$ may be identified with $j_{n}: B_{n}(\Omega X) \rightarrow B \Omega X$.

Moreover, since $G_{0}(X)=P X \simeq *$, any section $s$ to $g_{n}(X)$ satisfies $s \circ f \simeq q_{n}(X)$. Hence, we have ([13]) :

$$
\operatorname{cat}(X) \leq n \Longleftrightarrow g_{n}(X) \text { has a homotopy section }
$$

Remark $2 i$ ) Notice that with this definition, we do not need $A \rightarrow X$ to be a cofibration to define $\operatorname{cat}(X, A)$. Moreover, in this case, $\operatorname{cat}(X, A)$ is equal to the number $\operatorname{cat}(\tilde{X} ; A)$ obtained by turning $f$ into a cofibration $A \hookrightarrow \tilde{X}(\simeq X)$ and applying the covering definition of the relative category to the pair $(\tilde{X}, A)$.

For example, the numbers $\operatorname{cat}\left(X, S^{p} \times X\right)$ obtained by considering the projection $p r_{X}$ and $\operatorname{cat}\left(D^{p+1} \times X, S^{p} \times X\right)$ obtained by considering the inclusion $S^{p} \times X \hookrightarrow$ $D^{p+1} \times X$ are equal.

ii) The relative category is an invariant of the homotopy type of the pair.

Thus, one may easily see that $\operatorname{cat}\left(X, X \times S^{p}\right)=\operatorname{cat}\left(X, S^{p} \times X\right)=\operatorname{cat}\left(D^{p+1} \times\right.$ $\left.X, S^{p} \times X\right)=\operatorname{cat}\left(X \times D^{p+1}, X \times S^{p}\right)=\operatorname{cat}\left(X \times D^{p+1} \times D^{q+1}, X \times S^{p} \times D^{q+1}\right)$, where all the maps are the obvious ones.

iii) We may construct the relative Ganea fibration $g_{n+1}(X, A)$ directly as the join (see [6]) of the maps $g_{n}(X): G_{n}(X) \rightarrow X$ and $f: A \rightarrow X$.

Applying this to the particular case of the relative category of the projection $S^{p} \times X \rightarrow X$, we obtain the diagram

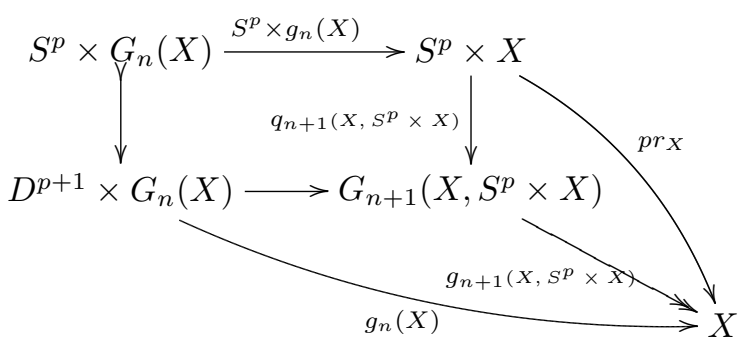

where $G_{n+1}\left(X, S^{p} \times X\right)$ is constructed as a push-out and the fibration $g_{n+1}\left(X, S^{p} \times\right.$ $X)$ is induced by $g_{n}(X)$ and $p r_{X}$.

Moreover, the fiber of $g_{n+1}\left(X, S^{p} \times X\right)$ can be obtained as push-out of the fibers of the other maps going to $X$, i.e. $F_{n+1}\left(X, S^{p} \times X\right)$ is the push-out of $S^{p} \times F_{n}(X) \hookrightarrow D^{p+1} \times F_{n}(X)$ and of $p r_{S^{p}}: S^{p} \times F_{n}(X) \rightarrow S^{p}$.

\subsubsection{Fiberwise Functorial Extension}

As mentionned in the introduction, many homotopical invariants related to the LS-category are defined by applying particular functors to the Ganea fibrations and by looking for sections to the maps thus obtained.

For example, the $\sigma^{i} \operatorname{cat}(X)$ (see [32]) is the smallest $n$ such that the $i$-fold suspension of the $n$-th Ganea fibration admits a section :

$$
\sigma^{i} \operatorname{cat}(X) \leq n \Longleftrightarrow \Sigma^{i} g_{n}(X): \Sigma^{i} G_{n}(X) \rightarrow \Sigma^{i} X \text { has a homotopy section }
$$


The invariant we are interested in is obtained by applying a fiberwise extension of (a base point free version of) $\Omega^{p} \Sigma^{p}$ to the Ganea fibrations.

Recall that a functor $\lambda: \mathcal{T} o p \rightarrow \mathcal{T}$ op is regular coaugmented if it sends contractible spaces to contractible spaces, preserves weak equivalences and is equipped with a natural transformation $\iota_{\lambda}: i d \rightarrow \lambda$ called the coaugmentation. Being given such a functor $\lambda$ and $p: E \rightarrow B$ a map with homotopy fiber $F$, we want to construct a space $\bar{\lambda}(E)$ and a map $p_{\bar{\lambda}}: \bar{\lambda}(E) \rightarrow B$ with homotopy fiber $\lambda(F)$ such that $p$ factors through $\bar{\lambda}(E)$.

We first detail an example of a fiberwise extension when $p$ is a fibration with connected fiber $F$ (see $[10]$ p.29). Since $F$ is connected, $p$ is the pullback of the universal fibration $F \rightarrow$ Baut $^{\bullet}(F) \rightarrow \operatorname{Baut}(F)$ over a classifying map $\phi: B \rightarrow \operatorname{Baut}(F)$. (Here, aut $(F)$ is the monoid of self-equivalences of $F$, aut $^{\bullet}(F)$ is the monoid of pointed self-equivalences of $F$ with respect to any choice of base point $* \in F$ and $B M$ denotes the classifying space for the monoid $M$.) Moreover, as $F$ has the homotopy type of a $C W$-complex, the coaugmentation $\iota_{F}: F \rightarrow \lambda(F)$ yields to a map $\operatorname{Baut}\left(\iota_{F}\right): \operatorname{Baut}(F) \rightarrow \operatorname{Baut}(\lambda(F))$ which induces a map between the corresponding universal fibrations. Then, the pull-back of $\lambda(F) \rightarrow$ Baut $^{\bullet}(\lambda(F)) \rightarrow \operatorname{Baut}(\lambda(F))$ over Baut $\left(\iota_{F}\right) \circ \phi$ is precisely a fibration with base space $B$ and fiber $\lambda(F)$. The arrow between $E$ and $\bar{\lambda}(E)$ is induced by the pull-back property.

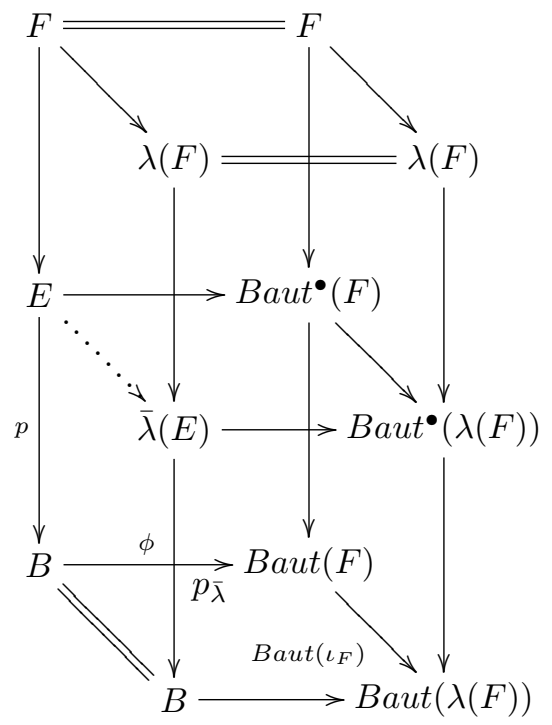

The precise fiberwise extension we use in the sequel is due to Dror Farjoun [10]. He shows that any regular coaugmented functor $\lambda$ admits a functorial fibrewise extension. More precisely, there exists a functor $\bar{\lambda}$ from the category of spaces over a space to itself such that for any map $p: E \rightarrow B$ we have a natural 
diagram

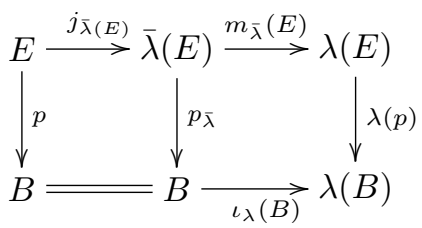

in which :

i) the left square is commutative,

ii) the right square is homotopy commutative by a natural homotopy,

iii) the composite $m_{\bar{\lambda}}(E) \circ j_{\bar{\lambda}}(E)$ coincides with the coaugmentation $\iota_{\lambda}(E)$.

$i v)$ the map induced by $j_{\bar{\lambda}}(E): E \rightarrow \bar{\lambda}(E)$ between the homotopy fibres of $p$ and $p_{\bar{\lambda}}$ over a point $b \in B$ is naturally equivalent to the coaugmentation $\iota_{\lambda}$.

Scheerer, Stanley and Tanré [27] apply this process to the Ganea fibrations and obtain a diagram

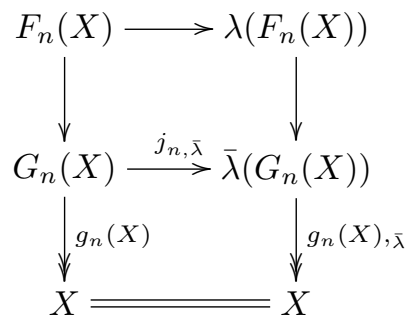

Since any section for $g_{n}(X)$ yields (by composition with $j_{n, \bar{\lambda}}$ ) to a section for $g_{n}(X), \bar{\lambda}$, they define in the usual way lower bounds for the LS-category.

Definition $4 \lambda$-cat $(X)$ is the least $n$ such that $g_{n}(X), \bar{\lambda}$ has a homotopy section.

\subsubsection{Q-category}

An important particular case of the general construction above and which is also discussed in [27] is the case of the functor $\Omega^{k} \tilde{\Sigma}^{k}$ (where $\tilde{\Sigma}^{k}$ denotes the $\mathrm{k}$-fold reduced suspension).

There are however some modifications needed. Indeed, $\Omega^{k} \tilde{\Sigma}^{k}$ is a pointed functor. Thus, to apply the construction of Dror Farjoun to $\Omega^{k} \tilde{\Sigma}^{k}$ and the Ganea fibrations one would need the fibers over each point of the base space to be pointed. This means that the respective fibrations must admit a section, which is not a priori the case.

Thus, in [27], Scheerer, Stanley and Tanré use a base point free version of the functor $\Omega^{k} \tilde{\Sigma}^{k}$ denoted by $Q^{k}$. This means that, contrary to $\Omega^{k} \tilde{\Sigma}^{k}$, the functor $Q^{k}$ is applied to spaces with no base point and that if a space $Z$ is pointed, then both spaces $\Omega^{k} \tilde{\Sigma}^{k} Z$ and $Q^{k}(Z)$ exist and are naturally homotopy equivalent. We do not give here the precise description of $Q^{k}$. However, we will give below (proof of Proposition 2) an equivalent construction of this functor.

For any $k \geq 0$ there exists a natural map $\Omega^{k} \tilde{\Sigma}^{k} Z \rightarrow \Omega^{k+1} \tilde{\Sigma}^{k+1} Z$ which is 
compatible with the coaugmentations.

We have the same situation in the base point free setting with a natural map $Q^{k}(Z) \rightarrow Q^{k+1}(Z)$ satisfying

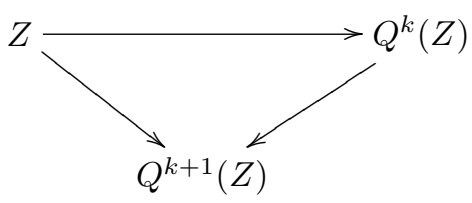

Using the Dror Farjoun construction, we get a commutative diagram

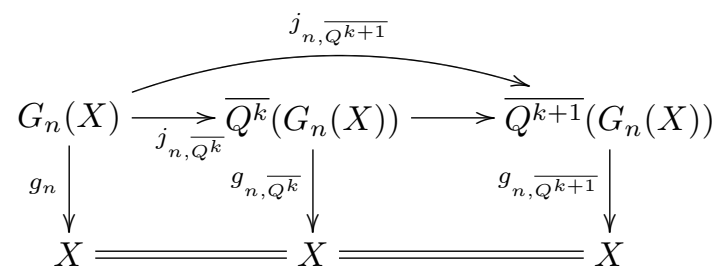

from which we deduce the inequalities

$$
\cdots \leq Q^{k+1} \operatorname{cat}(X) \leq Q^{k} \operatorname{cat}(X) \leq \cdots \leq Q^{1} \operatorname{cat}(X) \leq Q^{0} \operatorname{cat}(X)=\operatorname{cat}(X)
$$

We set $($ see $[33])$

Definition $5 \operatorname{cat}(X):=\lim _{k \rightarrow \infty} Q^{k} \operatorname{cat}(X)$.

Our aim is now to prove

Proposition 2 For any space $X$ of the homotopy type of a $C W$-complex,

$$
\operatorname{cat}\left(D^{p+1} \times X, S^{p} \times X\right)=Q^{p+1} \operatorname{cat}(X)+1
$$

Proof. For a space $Y$ and $p \in \mathbf{N}$, we define :

- $S^{p} * Y$ as the push-out of the following diagram and $\mu_{Y}: S^{p} \rightarrow S^{p} * Y$ as indicated below;

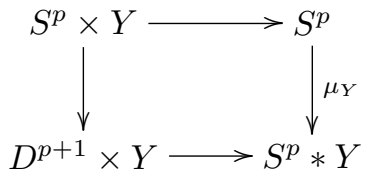

- $R^{p+1}(Y)=\left\{\omega: D^{p+1} \rightarrow S^{p} * Y\right.$ such that $\left.\omega_{\mid S^{p}}=\mu_{Y}\right\}$.

Remark that, using the exponential law in the above diagram, we get a map $\iota(Y): Y \rightarrow R^{p+1}(Y)$.

In the obvious way the construction $R^{p+1}$ turns out to be a coaugmented functor $\mathcal{T}$ op $\rightarrow \mathcal{T}$ op

Moreover, since for any pointed space $Y$ the map $\mu_{Y}: S^{p} \rightarrow S^{p} * Y$ is na- turally homotopically trivial and $S^{p} * Y \simeq \Sigma^{p+1} Y$ by a natural homotopy equivalence, this functor is a base point free version of $\Omega^{k+1} \tilde{\Sigma}^{k+1}$.

The functor $R^{p}$ turns out to be weakly equivalent as regular coaugmented functor to the functor $Q^{p}$ considered by Stanley, Scheerer, Tanré [27]. Thus, the invariants $R^{p}$ cat and $Q^{p}$ cat are equal. 
Now, our aim is to link the $R^{p+1}$-category with the relative category of the pair $\left(D^{p+1} \times X, S^{p} \times X\right)$.

In the following, for the sake of clarity, we will write $g_{n}$ for $g_{n}(X), g_{n+1}$ for $g_{n+1}\left(D^{p+1} \times X, S^{p} \times X\right)$ and $G_{n+1}(X, p)$ for $G_{n+1}\left(D^{p+1} \times X, S^{p} \times X\right)$.

Consider the following diagram (compare with [21], proof of Thm 2, Thm 3).

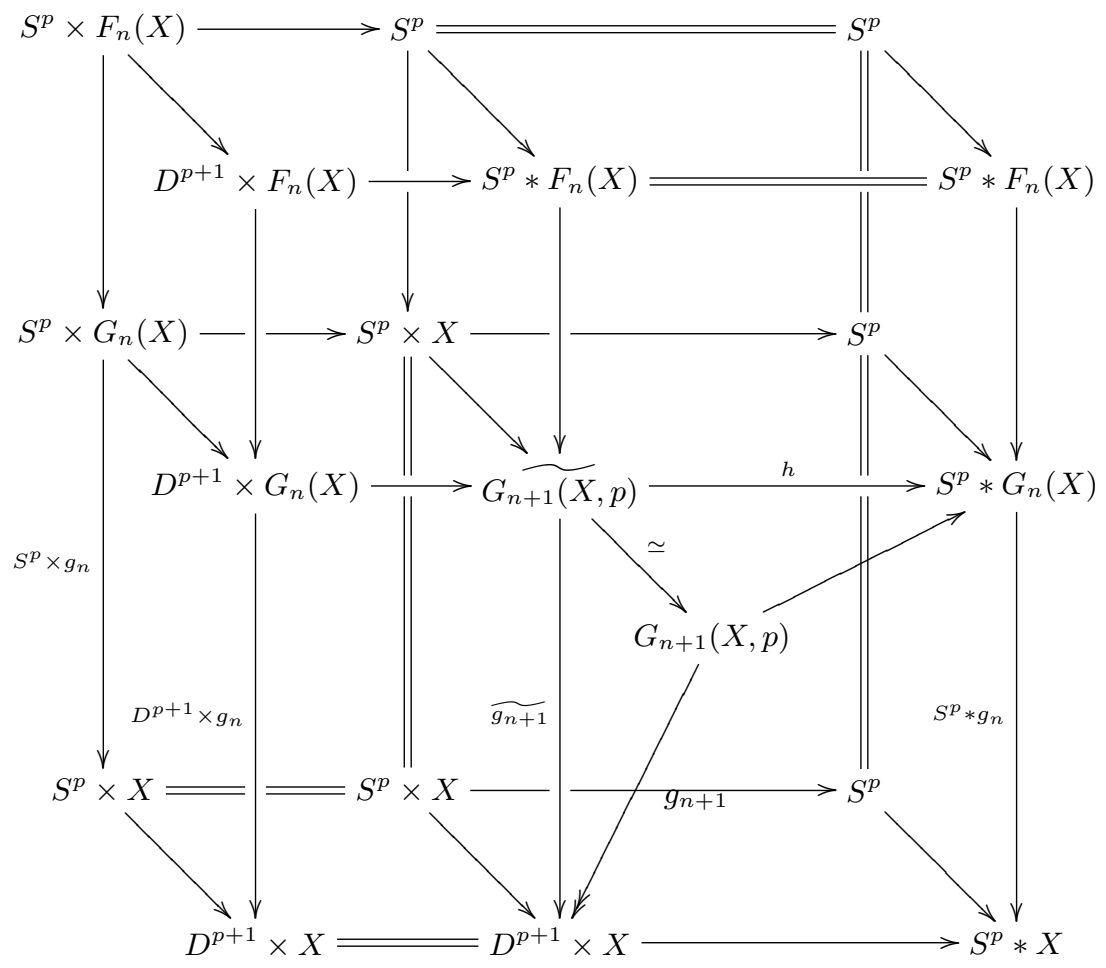

To obtain this diagram, we begin with the left and back faces and construct all the horizontal squares as push-out squares with induced map between them. Thus, the full diagram is the construction of $S^{p} * F_{n}(X) \rightarrow S^{p} * G_{n}(X) \rightarrow S^{p} * X$ and the left lower cube is a variant of the construction in the Remark $2 i i i)$. The map $\widetilde{g_{n+1}}$ is the induced map between the two push-out squares and we obtain $g_{n+1}$ by turning $\widetilde{g_{n+1}}$ into a fibration.

Indeed, $\widetilde{g_{n+1}}$ is not a fibration. However, composing the vertical maps of the left lower cube with the projection on $X$, we obtain fibrations whose fibers are represented in the upper left square. Thus, this upper square gives the construction of the homotopy fiber of $\widetilde{g_{n+1}}$ as push-out square.

The map $\left.\widetilde{G_{n+1}(X}, p\right) \rightarrow G_{n+1}(X, p)$ corresponds to the map $A \rightarrow \hat{A}$ in the beginning of section 2.2.1 . The map $G_{n+1}(X, p) \rightarrow S^{p} * G_{n}(X)$ is obtained as the homotopy equivalence $\left.G_{n+1}(X, p) \rightarrow \widetilde{G_{n+1}(X}, p\right)$ (corresponding to $\hat{A} \rightarrow A$ ) followed by $h$.

This diagram commutes exactly, except for the front "square" (between $G_{n+1}(X, p)$ and $\left.S^{p} * X\right)$ which is homotopy commutative by a natural homotopy relative to $S^{p} \times X$ 
Now, we have :

$$
\begin{aligned}
& \operatorname{cat}\left(D^{p+1} \times X, S^{p} \times X\right) \leq n \\
\Longleftrightarrow & g_{n+1}:\left(G_{n+1}(X, p), S^{p} \times X\right) \rightarrow\left(D^{p+1} \times X, S^{p} \times X\right)
\end{aligned}
$$

seen as a map of pairs, has a section

$s:\left(D^{p+1} \times X, S^{p} \times X\right) \rightarrow\left(G_{n+1}(X, p), S^{p} \times X\right)$.

Using the exponential law, we see that this is equivalent to associate to each $x \in X$ a map $\omega_{x}:\left(D^{p+1}, S^{p}\right) \rightarrow\left(G_{n+1}(X, p) ; S^{p} \times X\right)$ making the following diagram commutative

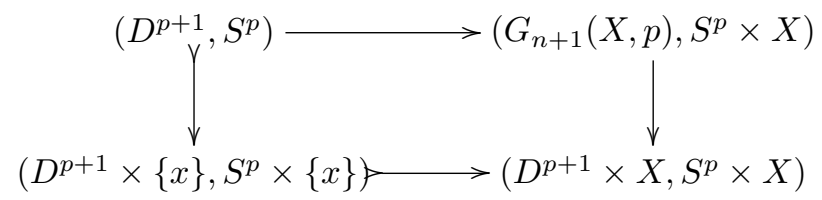

Define now

$$
R_{X}^{p+1}\left(G_{n+1}(X, p)\right)=\left\{\begin{array}{l}
\omega:\left(D^{p+1}, S^{p}\right) \rightarrow\left(G_{n+1}(X, p), S^{p} \times X\right) / \\
\exists x \in X \text { s.t. } \omega \text { makes the above diagram commutative }
\end{array}\right\}
$$

and remark that there is an obvious map $g_{n, R_{X}^{p+1}}: R_{X}^{p+1}\left(G_{n+1}(X, p)\right) \rightarrow X$.

Thus, we have

$$
\operatorname{cat}\left(D^{p+1} \times X, S^{p} \times X\right) \leq n+1 \Longleftrightarrow g_{n, R_{X}^{p+1}} \text { has a section }
$$

The middle left square of the big diagram represents a map of pairs $\left.\left(D^{p+1} \times G_{n}(X), S^{p} \times G_{n}(X)\right) \rightarrow\left(\widetilde{G_{n+1}(X}, p\right), S^{p} \times X\right)$ yielding to a map $\left(D^{p+1} \times G_{n}(X), S^{p} \times G_{n}(X)\right) \rightarrow\left(G_{n+1}(X, p), S^{p} \times X\right)$.

Then, by applying the exponential law, we get a map $\alpha_{X}^{p+1}: G_{n}(X) \rightarrow R_{X}^{p+1}\left(G_{n+1}(X, p)\right)$. Similarly, we have a map of pairs $\left(G_{n+1}(X, p), S^{p} \times X\right) \rightarrow\left(S^{p} * G_{n}(X), S^{p}\right)$ and the composition of an element of $R_{X}^{p+1}\left(G_{n+1}(X, p)\right)$ with this map is an element of $R^{p+1}\left(G_{n}(X)\right)$.

Thus, there is a map $\beta_{X}^{p+1}: R_{X}^{p+1}\left(G_{n+1}(X, p)\right) \rightarrow R^{p+1}\left(G_{n}(X)\right)$.

These maps fit into the diagram

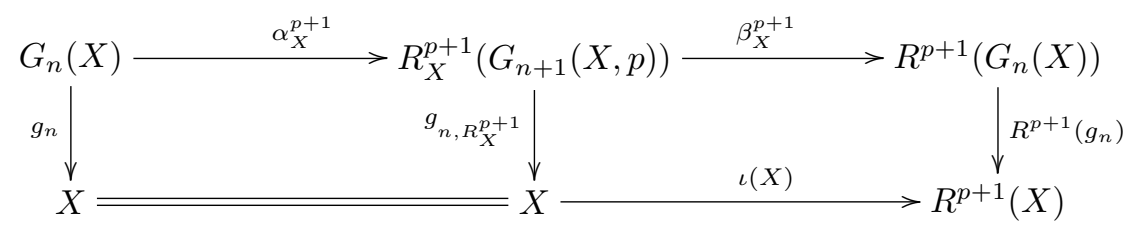

where the left square commutes exactly and the right square is homotopy commutative by a natural homotopy.

Moreover, by property of the exponential law $\beta_{X}^{p+1} \circ \alpha_{X}^{p+1}=\iota\left(G_{n}(X)\right)$, the map obtained by applying the exponential law to $\left(D^{p+1} \times G_{n}(X), S^{p} \times G_{n}(X)\right) \rightarrow$ $\left(S^{p} * G_{n}(X) ; S^{p}\right)$.

Finally, the map induced by $\alpha_{X}^{p+1}$ between the homotopy fibers of $g_{n}$ and $g_{n, R_{X}^{p+1}}$ is clearly the map obtained by applying the exponential law to $\left(D^{p+1} \times\right.$ 
$\left.F_{n}(X), S^{p} \times F_{n}(X)\right) \rightarrow\left(S^{p} * F_{n}(X) ; S^{p}\right)$, i.e. $\iota\left(F_{n}(X)\right)$.

Thus, by unicity of the fiberwise extension ([33], Prop. 2), $R_{X}^{p+1}\left(G_{n+1}(X, p)\right)$ $\rightarrow X$ is the fiberwise extension of $R^{p+1}$ applied to $g_{n}$.

That is, we may identify :

- $g_{n, R_{X}^{p+1}}$ with $g_{n, \overline{R^{p+1}}}: \overline{R^{p+1}}\left(G_{n}(X)\right) \rightarrow X$,

- $\alpha_{X}^{p+1}$ with $j_{n, \overline{R^{p+1}}}: G_{n}(X) \rightarrow \overline{R^{p+1}}\left(G_{n}(X)\right)$;

- $\beta_{X}^{p+1}$ with $m_{n, \overline{R^{p+1}}}: \overline{R^{p+1}}\left(G_{n}(X)\right) \rightarrow R^{p+1}\left(G_{n}(X)\right)$.

Thus,

$$
\begin{aligned}
\operatorname{cat}\left(D^{p+1} \times X ; S^{p} \times X\right) \leq n+1 & \Longleftrightarrow g_{n, R_{X}^{p+1}} \text { has a section } \\
& \Longleftrightarrow g_{n, \overline{R^{p+1}}} \text { has a section } \\
& \Longleftrightarrow R^{p+1} \operatorname{cat}(X) \leq n \\
& \Longleftrightarrow Q^{p+1} \operatorname{cat}(X) \leq n
\end{aligned}
$$

Hence the result.

We get now :

Corollary 2 For any space $X$ of the homotopy type of a $C W$-complex,

$$
\inf _{p \in \mathbf{N}} \operatorname{cat}\left(X \times D^{p+1}, X \times S^{p}\right)=Q \operatorname{cat}(X)+1
$$

\section{Final comments}

\subsection{Qcategory and other homotopy invariants}

In this section, all the spaces have the homotopy type of well-pointed $C W$ complexes. We compare the Qcategory with others homotopy invariants and provide some computations which illustrate our results.

The $\cup$-length of a space $X$ is the biggest $n$ such that there is a non-zero cup product of $n$ cohomology classes $u_{1}, \ldots, u_{n} \in \bar{H}^{*}(X)$. Thus, in this invariant, one considers implicitly that all the cohomology classes have the same "weight" 1. A more precise approach is given by the notion of (strict) category weight of a cohomology class [8], [24]. For $u \in H^{*}(X)$ the strict category weight of $u$ is defined by $\operatorname{swgt}(u)=\sup \left\{k \mid g_{k-1}^{*}(u)=0\right\}=\inf \left\{k \mid g_{k}^{*}(u) \neq 0\right\}$. Consider now the following definition [7] :

Definition 6 Let $X$ be a space and $\varphi: X \rightarrow K\left(\pi_{1}(X) ; 1\right)$ be the fundamental map, inducing an isomorphism on the fundamental groups.

Consider all the non-zero products $w=u_{\pi} \cup u_{1} \cup \ldots \cup u_{n} \in H^{*}(X)$ with $u_{\pi} \in$ $\varphi^{*}\left(H^{*}\left(K\left(\pi_{1}(X) ; 1\right)\right)\right)$ and $u_{i} \in \bar{H}^{*}(X)$ for $1 \leq i \leq n$ and $\operatorname{set} l(w)=\operatorname{dim}\left(u_{\pi}\right)+$ $n$.

Then, $\cup_{\pi}$ length $(X)$ is the maximum value of $l(w)$ for any such $w \in H^{*}(X)$

Clearly, $\cup$-length $(X) \leq \cup_{\pi}$ length $(X)$ and if $X$ is simply connected, then we take $u_{\pi}=1 \in H^{0}(X)$ and obtain $\cup_{\pi}$ length $(X)=\cup$-length $(X)$.

Moreover, extending an argument in the proof of Thm 4.1 [26], it is easy to see that, with the above notations, $\operatorname{swgt}\left(u_{\pi}\right)=\operatorname{dim}\left(u_{\pi}\right)$ and thus : 
$\operatorname{swgt}(w) \geq \operatorname{swgt}\left(u_{\pi}\right)+\operatorname{swgt}\left(u_{1}\right)+\ldots+\operatorname{swgt}\left(u_{n}\right) \geq \operatorname{dim}\left(u_{\pi}\right)+n=l(w)$.

We recall now the inequalities

$$
\cup_{\pi} \text { length }(X) \leq \sigma \operatorname{cat}(X) \leq Q \operatorname{cat}(X)
$$

The first inequality comes directly from [24], (2.5). Indeed, recall that $\sigma \operatorname{cat}(X)$ coincides with $r(X):=\sup _{u, E}\left\{\operatorname{swgt}(u) \mid u \in E^{*}(X), u \neq 0\right\}$ where $E$ runs over all cohomology theories. Then, if $w$ is a cohomological class such that $l(w)=$ $\cup_{\pi}$ length $(X)$, we have $\sigma \operatorname{cat}(X)=r(X) \geq \operatorname{swgt}(w) \geq l(w)=\cup_{\pi}$ length $(X)$.

The second inequality comes from [27] and can easily be recovered from proposition 2 and from [21].

Remark 3 To compute some examples of Qcategory, we recall the Theorem 15 of [33] :

Let $X$ be a $(r-1)$-connected $C W$-complex with $r$ cat $(X) \geq 3$. If $\operatorname{dim}(X) \leq$ 2.r.cat $(X)-3$ then $Q \operatorname{cat}(X)=\operatorname{cat}(X)$.

1) The symplectic group $S p(n)$ is a $n .(2 n+1)$-dimensional 2-connected manifold defined by $S p(n)=S p(n, \mathbf{C}) \cap U(2 n)$, where $S p(n, \mathbf{C})$ is the set of $2 n \times 2 n$ complex matrices $A$ satisfying $A^{t r} . J_{n} . A=J_{n}$ with $J_{n}=\left(\begin{array}{cc}0 & -I_{n} \\ I_{n} & 0\end{array}\right)$.

From [30], we have the inequalities $\cup$-length $(S p(n))=\cup_{\pi}$ length $(S p(n))=n<$ $\operatorname{cat}(S p(n))$. Thus, we easily get that $Q \operatorname{cat}(S p(2))=\operatorname{cat}(S p(2))(=3$ from [28]) and $\operatorname{Qcat}(\operatorname{Sp}(3))=\operatorname{cat}(\operatorname{Sp}(3))(=5$ from [11]).

2) Now, consider the case when $X=N \times T^{n}$. The space $N \times T^{n}$ is 0 -connected $(\mathrm{r}=1)$, has dimension $\operatorname{dim}(N)+n$ and 2.cat $\left(N \times T^{n}\right)-3 \geq 2 . \operatorname{cat}\left(T^{n}\right)-3 \geq 2 . n-3$. Thus, for $n$ big enough, (e.g. $n \geq \operatorname{dim}(N)+3), \operatorname{Qcat}\left(N \times T^{n}\right)=\operatorname{cat}\left(N \times T^{n}\right)$ (compare with [23]).

3 ) We do not know examples of closed manifolds with $Q$ cat $\neq \sigma c a t$. However, the two invariants usually differ as seen on the following example. Consider the Poincaré sphere $Z^{3}$, obtained as quotient of $S^{3}$ by the binary icosahedral group, a perfect group of order $120 . Z^{3}$ is a closed manifold of dimension 3 and from [15] $\operatorname{cat}\left(Z^{3}\right)=3$. Using the theorem above, we also have $Q \operatorname{cat}\left(Z^{3}\right)=3$. Now, consider $A=Z^{3}-D^{3}$, the Poincaré sphere with a small disk removed. $A$ is not contractible since $\pi_{1}(A)=\pi_{1}\left(Z^{3}\right)$ but is acyclic. Hence, $\Sigma A \simeq *$ and thus $\sigma \operatorname{cat}(A)=0$. However, $Q \operatorname{cat}(A)=\operatorname{cat}(A)=2$. Indeed, $A$ is a $C W$-complex of dimension 2. Hence, $Q \operatorname{cat}(A) \leq \operatorname{cat}(A) \leq 2$. Moreover, $Z^{3}=A \amalg_{S^{2}} D^{3}$. Thus, by $[27], 3=Q \operatorname{cat}\left(Z^{3}\right) \leq Q \operatorname{cat}(A)+1$.

Notice that there also exist 1-connected CW-complexes for which $\sigma c a t \neq Q c a t$ (see $[27])$.

\subsection{Lagrangian Intersections in Cotangent Bundles}

Let $(V, \omega)$ be a symplectic manifold (i.e $V$ is an even-dimensional smooth manifold and $\omega$ is a closed non-degenerate 2-form on $T V$ ).

A Lagrangian submanifold of $V$ is a submanifold $L$ satisfying $\omega_{\mid T L} \equiv 0$ and $\operatorname{dim}(L)=\frac{1}{2} \operatorname{dim}(V)$.

Given $H: V \times S^{1} \rightarrow \mathbf{R}$ a smooth function with compact support we define a family of vector fields $X_{H_{t}}$ by $\omega\left(X_{H_{t}},-\right)=-d H_{t}$.

Now, for each $v \in V$, consider the differential equation $\left\{\begin{aligned} \frac{d}{d t} x(t) & =X_{H_{t}}(x(t)) \\ x(0) & =v\end{aligned}\right.$ 
and set $\varphi^{t}(v)=x(t)$.

$\left(\varphi^{t}\right)$ thus defined is a 1-parameter family of diffeomorphisms, called a hamiltonian isotopy, and $\psi=\varphi^{1}$ is called a hamiltonian diffeomorphism.

Given $V, L$ and $\psi$ as above, a classical problem in symplectic topology is to study the intersection $\psi(L) \cap L$.

Recall now that if $M$ is a closed, smooth manifold, then the cotangent bundle $T^{*} M$ is a symplectic manifold with symplectic form $\omega=-d \lambda$, where $\lambda$ is the Liouville form. Moreover, $M$ viewed as the zero section, is a Lagrangian submanifold of $T^{*} M$.

In this particular case, the Arnold Conjecture may be stated as :

Conjecture : Let $M$ be a closed manifold. For any hamiltonian diffeomorphism with compact support $\psi$ of $T^{*} M$,

$$
\#(\psi(M) \cap M) \geq \operatorname{crit}(M)
$$

A way to approach this problem is to replace in the inequality $\operatorname{crit}(M)$ by numerical homotopy invariants known to be lower bounds for $\operatorname{crit}(M)$. For example, Hofer [16] proves that \# $(\psi(M) \cap M) \geq \cup$-length $(M)+1$.

Another approach is due to Laudenbach and Sikorav [19]. Using the method of "broken geodesics" developped by Chaperon [1], they prove the inequality $\#(\psi(M) \cap M) \geq \widetilde{\operatorname{crit}}(M)$. This result has been generalized by Eliashberg and Gromov [7], using the method of generating functions (see [29]). They also give as corollary the inequality $\#(\psi(M) \cap M) \geq \cup_{\pi}$ length $(M)+1$.

Since $\operatorname{Qcat}(M) \geq \cup_{\pi}$ length $(M) \geq \cup$-length $(M)$, the aforementioned inequalities of [16] and [7] are obtained as corollaries. As remarked in section 3.1, the strict inequality $\operatorname{Qcat}(M)>\cup_{\pi}$ length $(M)$ may occur.

At last, using the remark 3.2, we may restate Corollary $B$ in the particular case of the products $N \times T^{n}$ as :

Let $N$ be a closed manifold and take $n \geq \operatorname{dim}(N)+3$. Then, for any hamiltonian diffeomorphism with compact support $\psi$ of $T^{*}\left(N \times T^{n}\right)$,

$$
\#\left(\psi\left(N \times T^{n}\right) \cap\left(N \times T^{n}\right)\right) \geq \operatorname{cat}\left(N \times T^{n}\right)+1
$$

This last inequality is the best possible from a homotopical point of view.

\subsection{On $\operatorname{crit}(M)$ and $\widetilde{\operatorname{crit}}(M)$}

A way to study $\operatorname{crit}(M)$ is by studying its approximations by homotopy invariants. Another lower bound for $\operatorname{crit}(M)$ is given by $\widetilde{\operatorname{crit}}(M)$. It is not clear if $\widetilde{\operatorname{crit}}(M)$ is a homotopy invariant but it seems closely linked to the homotopical properties of $M$.

In [4], if $M$ is a 2-connected $n$-dimensional manifold, Cornea constructs on $M \times D^{n+1}$ a function $f$ with at most $\operatorname{cat}(M)+2$ critical points, whose gradient flow points inward on $M \times S^{n}$. Using a partition of unity, it is easy to extend $f$ on $M \times \mathbf{R}^{n+1}$ by a quadratic form of index $n$ (without adding any new critical points). Thus, we get a function quadratic at infinity with at most $\operatorname{cat}(M)+2$ critical points. This result is valid, in fact, for 1-connected manifolds [5].

Combining this with the corollary 1, we get

Corollary 3 For any closed 1-connected manifold,

$$
\operatorname{Qcat}(M)+1 \leq \widetilde{\operatorname{crit}}(M) \leq \operatorname{cat}(M)+2
$$


To be more precise, the function $f$ described above has in fact $C l_{F}(M)$ critical points, where $C l_{F}(M)$ is the least number of stages needed to construct a space homotopic to $M$ by attaching cones over finite $C W$-complexes. $C l_{F}$ is a homotopy invariant satisfying $c a t \leq C l_{F} \leq c a t+1$.

Thus, for any manifold $M$ satisfying $Q \operatorname{cat}(M)=C l_{F}(M)$ (which is a purely homotopical condition), we have $\widetilde{\operatorname{crit}}(M)=\operatorname{cat}(M)+1$. Moreover, in this case, for any manifold $N$ homotopic to $M$ we have $\widetilde{\operatorname{crit}}(M)=\widetilde{\operatorname{crit}}(N)$.

These inequalities also allow some computations.

For example, since $Q \operatorname{cat}(S p(2))=C l_{F}(S p(2))=3, \widetilde{\operatorname{crit}}(S p(2))=3+1=4$.

Similarly, $Q \operatorname{cat}(S p(3))=C l_{F}(S p(3))=5$ (see [11]) and thus $\widetilde{\operatorname{crit}}(\operatorname{Sp}(3))=6$.

Remark 4 The question remains of the possibility to enhance the result Qcat+ $1 \leq \widehat{\text { crit }}$ by replacing Qcat by a bigger homotopy invariant.

Recently, Razvan [22] using a variant of the relative category, claimed a result leading to $c a t+1 \leq \widetilde{\text { crit }}$. Unfortunately, as we shall see below, there is a serious mistake in his argument and the question of whether this last inequality holds or not remains open.

He defines $\widetilde{c a t}(X ; A)$ as the smallest $k$ such that $X$ may be covered by $k+1$ open sets $\left(U_{i}\right)_{0 \leq i \leq k}$ such that $U_{0}$ can be deformed into $A$ (rel. $A$ ) and the other open sets $U_{i} \subset X-A$ are contractible in $X-A$ and proves that in particular $\operatorname{cat}\left(X \times D^{n+1} ; X \times S^{n}\right) \geq \operatorname{cat}(X)+1$.

However, if $A \rightarrow X$ is a closed cofibration, then we clearly see that $\operatorname{cat}(X ; A)$ $=\widetilde{c a t}\left(X \cup_{A \times\{0\}} A \times I ; A \times\{1\}\right)$. Thus, for any manifold $N$ with boudary, using the collar theorem, we get $\operatorname{cat}(N ; \partial N)=\widetilde{\operatorname{cat}}(N ; \partial N)$. In particular, if $M$ is a closed manifold, $\operatorname{cat}\left(M \times D^{n+1} ; M \times S^{n}\right)=\widetilde{\operatorname{cat}}\left(M \times D^{n+1} ; M \times S^{n}\right)$.

Thus, Razvan's result leads to $\operatorname{cat}\left(M \times D^{n+1} ; M \times S^{n}\right) \geq \operatorname{cat}(M)+1$ which is not true in this generality (see section 3.4 below). In fact, in his paper the argument on page 53 1.-7 is mistaken as the sets $\pi\left(U_{i}\right)$ need not be contractible.

\subsection{Homotopical conjectures on numerical invariants}

In [3], Cornea formulated a conjecture claiming that, for $n$ big enough,

$$
\text { (CO) } \quad: \quad \operatorname{cat}\left(X \times D^{n+1} ; X \times S^{n}\right)=\operatorname{cat}(X)+1
$$

Because of the inequalities $\operatorname{cat}\left(X \times D^{n+1} ; X \times S^{n}\right) \leq \operatorname{cat}\left(X \times S^{n}\right) \leq \operatorname{cat}(X)+1$, $(\mathrm{CO})$ implied $\operatorname{cat}\left(X \times S^{n}\right)=\operatorname{cat}(X)+1$, the Ganea Conjecture.

It is now known that the Ganea conjecture is not true [17] and therefore (CO) can not hold either for all spaces $X$. However, using proposition 2, we get

$$
\mathrm{X} \text { satisfies }(\mathbf{C O}) \Longleftrightarrow Q \operatorname{cat}(X)=\operatorname{cat}(X)
$$

Since the equality $Q \operatorname{cat}(X)=\operatorname{cat}(X)$ has been proved in [27] when $X$ is a rational space, we have

Corollary 4 The Conjecture (CO) holds for rational spaces

Moreover, it was conjectured in [27] that

$$
Q \operatorname{cat}(X)=\operatorname{cat}(X) \Longleftrightarrow X \text { satisfies the Ganea Conjecture }
$$

At first, notice that the $\Rightarrow$ part of the equivalence is obtained as consequence of proposition 2 (and was proved independantly in [33] by means of a product 
formula for Qcat).

Moreover, if this conjecture holds, then the spaces for which $Q \operatorname{cat}(X) \neq \operatorname{cat}(X)$ are exactly the spaces not satisfying the Ganea conjecture.

There are very few such counter-examples (even if there are manifolds among them [18]) and therefore, at the moment, there are very few examples of closed manifolds with $Q$ cat $\neq$ cat.

Acknowledgement: The authors wish to thank O. Cornea and D. Tanré for many discussions, suggestions and comments. They also wish to thank the organizers of the Barcelona 2001 EuroPhD Topology Conference during which their collaboration began. The second author acknowledges the support of the Portuguese Foundation for Science and Technology (FCT) through the research program POCTI.

\section{References}

[1] M. Chaperon, Une idée du type "géodésiques brisées" pour les systèmes hamiltoniens, C. R. Acad. Sci. Paris Sér. I Math. 298 (1984), 293-296.

[2] C.C. Conley, E. Zehnder The Birkhoff-Lewis fixed point theorem and a conjecture of V.I. Arnold, Invent. Math. 73 (1983), 33-49.

[3] O. Cornea, Some properties of relative L.S.-category, Amer. Math. Soc., The Fields Institute for Research in Mathematical Sciences Communications 19 (1998), 67-72.

[4] O. Cornea, Cone-decompositions and degenerate critical points, Proc. London Math. Soc. 77 (1998), 437-461.

[5] O. Cornea, G. Lupton, J. Oprea, D. Tanre, Monograph on the LS-category, in preparation.

[6] J.-P. Doeraene, Homotopy pull backs, homotopy push outs and joins, Bull. Belg. Math. Soc. Simon Stevin 5 (1998), 15-37.

[7] Y. Eliashberg, M. Gromov Lagrangian intersection theory: finitedimensional approach, Amer. Math. Soc. Transl. Ser. 2186 (1998), 27-118.

[8] E. Fadell, S. Husseini, Category weight and Steenrod operations, Bol. Soc. Mat. Mexicana 37 (1992), 151-161.

[9] E. Fadell, S. Husseini, Relative category, products and coproducts, Rend. Sem. Mat. Fis. Milano 64 (1994), 99-115.

[10] E. Dror Farjoun, Cellular spaces, null spaces and homotopy localization, LNM 1622, Springer Verlag, 1996.

[11] L. Fernandez, A. Gomez, J. Strom, D. Tanré, The Lusternik-Schnirelmann category of $S p(3)$, Preprint 2001 to appear in the Proceedings of the AMS.

[12] A. Floer, Cuplength estimates on Lagrangian intersections, Comm. Pure Appl. Math. 42 (1989), 335-356. 
[13] T. Ganea, Lusternik-Schnirelmann category and strong category, Illinois. J. Math. 11 (1967), 417-427.

[14] T. Ganea, A generalization of the homology and homotopy suspension, Comment. Math. Helv. 39 (1964), 295-322.

[15] J.C. Gomez-Larranaga, F. Gonzales-Acuna, Lusternik-Schnirelmann category of 3-manifolds, Topology 31 (1992), 791-800.

[16] H. Hofer, Lagrangian embeddings and critical point theory, Ann. Inst. H. Poincaré Anal. Non Linéaire 2 (1985), 407-462.

[17] N. Iwase, Ganea's conjecture on Lusternik-Schnirelmann category, Bull. London Math. Soc. 6 (1998), 623-634.

[18] N. Iwase, Spheres bundles over spheres, Preprint 2001.

[19] F. Laudenbach, J.-C. Sikorav, Persistance d'intersection avec la section nulle au cours d'une isotopie hamiltonienne dans un fibré cotangent, Invent. Math. 82 (1985), 349-357.

[20] W. Meyer, Kritische Mannigfaltigkeiten in Hilbertmannigfaltigkeiten, Math. Ann. 170 (1967), 45-66.

[21] P.-M. Moyaux, Lower bounds for the relative Lusternik-Schnirelmann category, Manuscripta Math. 101 (2000), 533-542.

[22] M. Razvan, Relative Ljusternik-Schnirelmann category of index pairs and applications to critical point theory, Proceedings of the 31st Iranian Mathematics Conference (Tehran, 2000), Univ. Tehran, (2000), 50-55.

[23] Y. Rudyak, On the Ganea conjecture for manifolds, Proc. Amer. Math. Soc. 125 (1997), 2511-2512.

[24] Y. Rudyak, On category weight and its applications, Topology 38 (1999), $37-55$.

[25] Y. Rudyak, On analytical applications of stable homotopy (the Arnold conjecture, critical points), Math. Z. 230 (1999), 659-672.

[26] Y. Rudyak, J. Oprea, On the Lusternik-Schnirelmann category of symplectic manifolds and the Arnold conjecture, Math. Z. 230 (1999), 673-678.

[27] H. Scheerer, D. Stanley, D. Tanré, Fiberwise construction applied to Lusternik-Schnirelmann category, To appear in Israel J. Math.

[28] P. Schweitzer, Secondary cohomology operations induced by the diagonal mapping, Topology 3 (1965), 337-355.

[29] J.-C. Sikorav, Problèmes d'intersections et de points fixes en géométrie hamiltonienne, Comment. Math. Helv 62 (1987), 62-73.

[30] W. Singhof, On the Lusternik-Schnirelmann category of Lie groups. II, Math. Z. 151 (1976), 143-148. 
[31] F. Takens, The minimal number of critical points of a function on a compact manifold and the Lusternik-Schnirelman category, Invent. Math. 6 (1968), 197-244.

[32] L. Vandembroucq, Suspension of Ganea fibrations and a Hopf invariant, Topology Appl. 105 (2000), 187-200.

[33] L. Vandembroucq, Fibrewise suspension and Lusternik-Schnirelmann category, Topology, 41 (2002), 1239-1258. 